\title{
A STUDY TO ASSESS THE KNOWLEDGE REGARDING ECG INTERPRETATION DURING CARDIAC EMERGENCIES AMONG THE STAFF NURSES OF SELECTED CARDIAC HOSPITALS OF BATHINDA IN PUNJAB
}

\author{
Mr. Nibu G. Philip* I Dr. Krishna Chauhan** \\ * Research Scholar, Himalayan University, Itanagar, Arunachal Pradesh, India. \\ ** Principal, Shimla Nursing College, Annadale, Himachal Pradesh, India. \\ DOI: http://doi.org/10.47211/trr.2020.v06i01.007 \\ Received $10^{\text {th }}$ April 2020, Accepted $25^{\text {th }}$ May 2020, Available online $25^{\text {th }}$ June 2020.
}

\begin{abstract}
Electrocardiogram (ECG) interpretation by nurses is a valuable tool for the initial evaluation of patients with cardiac problems which may lead to potentiallylife threatening complications. Nurses usually being the first ones to handle the ECG made them to be more creative to take appropriate decisions to save a life especially in critical units of hospital. Hence there is a need to increase the knowledge of the nurses on ECG interpretation to provide right intervention and to avoid complications. The aim of the study is to determine the knowledge in ECG interpretation during cardiac emergencies among the staff nurses. This descriptive study was conducted on the registered nurses who worked at selected cardiac hospitals at Bathinda. A total of 70 staff nurses were selected who voluntarily consented to be the sample of the study. A structured questionnaire that was developed by the researcher on the basis of the literature was used as data collection tool. Data were collected using a questionnaire after obtaining the ethical approval. Descriptive statistics and Pearson Chi-Square tests were used in analysing the data. Results of the study showed a good level of knowledge among nurses, regarding the ECG interpretation during cardiac emergencies. Working unit in hospital and previous ECG training courses play an importantrole in defining the professionalism of nurses to have a good experience in ECG interpretation. Training courses for nurses under the supervision of qualified staff especially for nurses who work in critical care area in hospital, with continuing self learning programmes and staying up to date to any changes and development of new protocols or technology help increase patient outcomes.
\end{abstract}

Key Words: Electrocardiogram, Knowledge, Interpretation, Cardiac Disorders

\section{ABOUT AUTHORS}

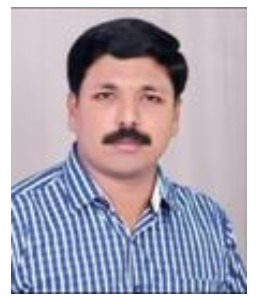

Author Mr. Nibu G Philip is a Research Scholar at Himalayan University in Itanagar, Arunachal Pradesh, India. He has attended various National and International conferences and workshops.

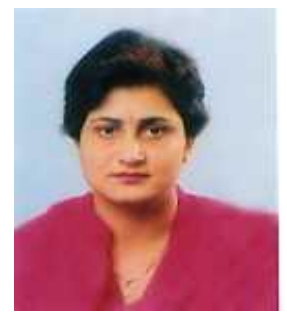

Author Dr. Krishna Chauhan is the Principal at Shimla Nursing College, Shimla. She has presented papers in various conferences and also has many publications to her name. 


\section{INTRODUCTION}

Electrocardiography is a commonly used non-invasive procedure for recording electrical changes in the heart. An electrocardiogram depicts the series of waves that relate to the electrical impulses which occur during each beat of the heart. Currently ECG is an essential evaluation tool for patients with cardiac problems, and it is considered as the first diagnostic tool in chest pain and it provides reliable information about the structure and function of the heart. ${ }^{1}$ Also ECG makes a focal point of modern medicine because it gives the whole background about diagnosing acute coronary syndromes and cardiac arrhythmias. ${ }^{2}$

According to World Health Organization, ischemic heart disease and stroke takes the first position globally among the top 10 causes of death, which accounted for a combined 15 million deaths in the year $2015 .^{3}$ In the health care centres nurses are usually the first responders to an in-hospital cardiac arrest and they must be master in basic resuscitation skills. ${ }^{4}$ Every nurse should be able to recognise basic ECG rhythms, such as normal sinus rhythm, sinus tachycardia, sinus bradycardia, atrial fibrillation, atrial flutter, heart blocks, ventricular fibrillation and asystole. ${ }^{5}$ It is required that the nurse must be responsible for monitoring and making clinical decisions based on information obtained from the monitor. $^{6}$

The nurses are accountable in continuous monitoring especially in critical care units, to ensure ongoing safe and effective ECG monitoring and to determine the actions required to improve the quality of care. ${ }^{7}$ Result of inappropriate interpretation increases the cost of healthcare and can delay the admission process which will be an unpleasant burden for the hospital and its patients. ${ }^{8}$

Rapid Access Chest Pain Clinic (RACPC) was established in England that helped the nurses to assess the patient and to plan the care immediately without waiting for a physician; and also an ECG is recorded immediately as the patient arrives at the clinic with chest pain, and the nurse consultant then examines and decides if further investigation is required. ${ }^{9}$ Many methods had been designed like CRISP (Cardiac Rhythm Identification for Simple People) which helps nurses to rapidly interpret ECG. ${ }^{5}$ Also American Heart Association designed a PULSE trial (Practical Use of the Latest Standards for Electrocardiography trial) which is an online ECG monitoring education programme and strategies to implement and sustain change in practice, led by nurse champions on each unit and this trial increases nurses' knowledge of ECG monitoring, quality of care related to ECG monitoring and the patient outcomes. ${ }^{10}$

\section{NEED FOR THE STUDY}

The educated and qualified nurses play an important role in preventing the complications that may occur to patients who have arrhythmias. Difficulties in interpreting the ECG of the patients with Acute Myocardial Infarction (AMI) with Left Bundle Branch Block (LBBB) may delay the treatment and affects their prognosis. ${ }^{11}$

American heart association (AHA) reveals that there is a prevalence of $11.5 \%$ of American adults (27.6 million) been diagnosed with heart disease and mortality is increasing every year since 1919. Coronary artery disease is accounted for more deaths than any other major cause of death in the United States. ${ }^{12}$ Among Indian women, CAD continues to be a major public health problem that represents a leading cause of death and disability. ${ }^{13}$ Also the presence of diabetes, hypertension, high levels of total cholesterol, and low levels of high-density lipoprotein, low-density lipoprotein and triglycerides all are correlated with CAD. ${ }^{14}$

A proper education and training play an important role in being qualified in interpreting the ECG. A study conducted among the staff nurses of CCU and ICU departments of Benha University hospital in Egypt showed that the nurses' performance in recording a 12-lead ECG and early detection of dysarrhythmia had improved after implementation of a training programme. ${ }^{15}$

Nurses working in ICU and emergency department are highly responsible for determination and use of applications for early detection and diagnosing the signs and symptoms of the patients. There is a need to increase the knowledge and practice of nurses on electrocardiogram to provide a better health care outcome for the patient and to avoid any mistake in ECG interpretation and interventions. Improvement of knowledge and practice of nurses on electrocardiogram maybe useful in improving their performance level and to make them initiated to become more professional in interpretation of ECG.

\section{PROBLEM STATEMENT}

'A study to assess the knowledge regarding ECG interpretation during cardiac emergencies among the staff nurses of selected cardiac hospitals of Bathinda in Punjab. 


\section{ARTICLES}

\section{OBJECTIVES}

1. To assess the knowledge regarding ECG interpretation during cardiac emergencies among the staff nurses at selected cardiac hospitals in Bathinda.

2. To determine the association between the socio-demographic variables of the staff nurses and their knowledge level regarding ECG interpretation during cardiac emergencies.

\section{RESEARCH METHODOLOGY}

Research Approach: A descriptive approach was used for the present study since the purpose of the study was to assess the knowledge level and its association with the selected variables.

Research Design: A descriptive design was used in this study.

Setting of the Study: The study was conducted at selected cardiac hospitals of Bathinda district in Punjab.

Population: For the present study target population is the staff nurses working at selected cardiac hospitals of Bathinda district in Punjab.

Sample and Sampling Technique -

Sample: The sample for the present study consists of staff nurses working at the selected cardiac hospitals of Bathinda district.

Sampling Technique: Purposive sampling was utilised for the selection of subjects.

Sample Size: 70 staff nurses were chosen to be the part of the study.

\section{Development of the Tool -}

Data collection procedures: The investigator personally visited each respondent and explained the purpose of the study. The respondents were assured anonymity and confidentiality of the information provided by them and an informed consent was obtained. Interviews were conducted during their free time. Data was collected with the help of the socio-demographic profile and a knowledge questionnaire. The data collection process was terminated after thanking participants for their participation and co-operation.

Description of the data collection tool: In this study the data collection tools consisted of 2 parts covering the following areas -

I. Socio-demographic data: It contains six (6) questions selected on background factors such as age, gender, qualification, present departmental experience, total years of experience and exposure to ECG training program.

II. Preparation of knowledge questionnaire: Knowledge questionnaire consisted of twenty-five (25) items regarding interpretation of life threatening arrhythmias and their management.

\section{METHOD OF DATA ANALYSIS:}

- Description of sample characteristics, Baseline data containing sample characteristics (socio-demographic data) was analysed using frequency and percentage.

- The knowledge of the nurses was analysed using frequency, percentage, mean percentage and standard deviation.

- Association between knowledge scores and selected demographic data was found out by using Chi-Square test. 


\section{ARTICLES}

\section{RESULTS}

Frequency and percentage distribution of demographic variables

Table - 1: Distribution of staff nurses of the selected Cardiac hospitals according to socio-demographic variables

$(n=70)$

\begin{tabular}{|c|c|c|c|}
\hline \multicolumn{2}{|c|}{ Variables } & \multirow{2}{*}{$\begin{array}{c}\text { No of respondents } \\
15\end{array}$} & \multirow{2}{*}{$\frac{\text { Percentage }}{21.4}$} \\
\hline Age in years & $21-25$ years & & \\
\hline & $26-30$ years & 25 & 35.7 \\
\hline & $31-35$ years & 16 & 22.8 \\
\hline & 36 and above & 14 & 20.0 \\
\hline \multirow[t]{2}{*}{ Gender } & Male & 15 & 21.4 \\
\hline & Female & 55 & 78.5 \\
\hline \multirow[t]{4}{*}{ Professional Qualification } & GNM & 25 & 35.7 \\
\hline & BSc Nursing & 25 & 35.7 \\
\hline & Post BSc Nursing & 20 & 28.5 \\
\hline & MSc Nursing & 00 & 00.0 \\
\hline \multirow[t]{4}{*}{ Present Departmental Experience } & Coronary Care Unit CCU & 20 & 28.5 \\
\hline & Emergency Department & 20 & 28.5 \\
\hline & CTCVS & 15 & 21.4 \\
\hline & Cath Lab & 15 & 21.4 \\
\hline \multirow[t]{4}{*}{ Years of Experience } & Less than 1 Year & 14 & 20.0 \\
\hline & $1-2$ Years & 25 & 35.7 \\
\hline & $3-4$ Years & 20 & 28.5 \\
\hline & Greater than 5 Years & 11 & 15.7 \\
\hline \multirow{2}{*}{$\begin{array}{l}\text { Exposure to ECG } \\
\text { Programme }\end{array}$} & Yes & 40 & 57.1 \\
\hline & No & 30 & 42.8 \\
\hline
\end{tabular}

The above table depicts that the majority $35.7 \%$ of the nurses was of $26-30$ years of age, and $22.8 \%$ among them were of $31-35$ years old, $21.4 \%$ were in the age group of $21-25$ years and the minority $20 \%$ was in the age group of above 36 years. In regard to their gender, majority $78.5 \%$ of the samples were females and the rest $21.4 \%$ were males. Considering their qualifications, $35.7 \%$ were GNM, 35.7\% of them were BSc Nurses, $28.5 \%$ were Post BSC Nurses and none were MSc Nurses. In respect of the present departmental experience $28.5 \%$ each was from the Coronary Care Unit and emergency department while 21.4\% each were from CTCVS and Cath Lab. As regards their years of experience, $20 \%$ had less than one year of experience and $35.7 \%$ were with experience of $1-2$ years, $28.5 \%$ with an experience of $3-4$ years and $15.7 \%$ had an experience of more than 5 years. $57.1 \%$ of the nurses had attended ECG training programme while $40 \%$ had no such exposure. 


\section{ARTICLES}

Table - 2: Distribution of knowledge scores regarding ECG interpretation during cardiac emergencies among the staff nurses

\begin{tabular}{|c|l|c|c|}
\hline SI. No & Level of knowledge & No. of Staff Nurses & Percentage \\
\hline 1. & Poor knowledge & 09 & 00 \\
\hline 2. & Average knowledge & 26 & 88.88 \\
\hline 3. & Good knowledge & 35 & 00 \\
\hline 4. & Very good knowledge & 70 & 11 \\
\hline
\end{tabular}

The above table reveals that the majority of the staff nurses $50 \%$ had good knowledge, $37.1 \%$ had average knowledge and $12.8 \%$ had poor knowledge regarding ECG interpretation during cardiac emergencies.

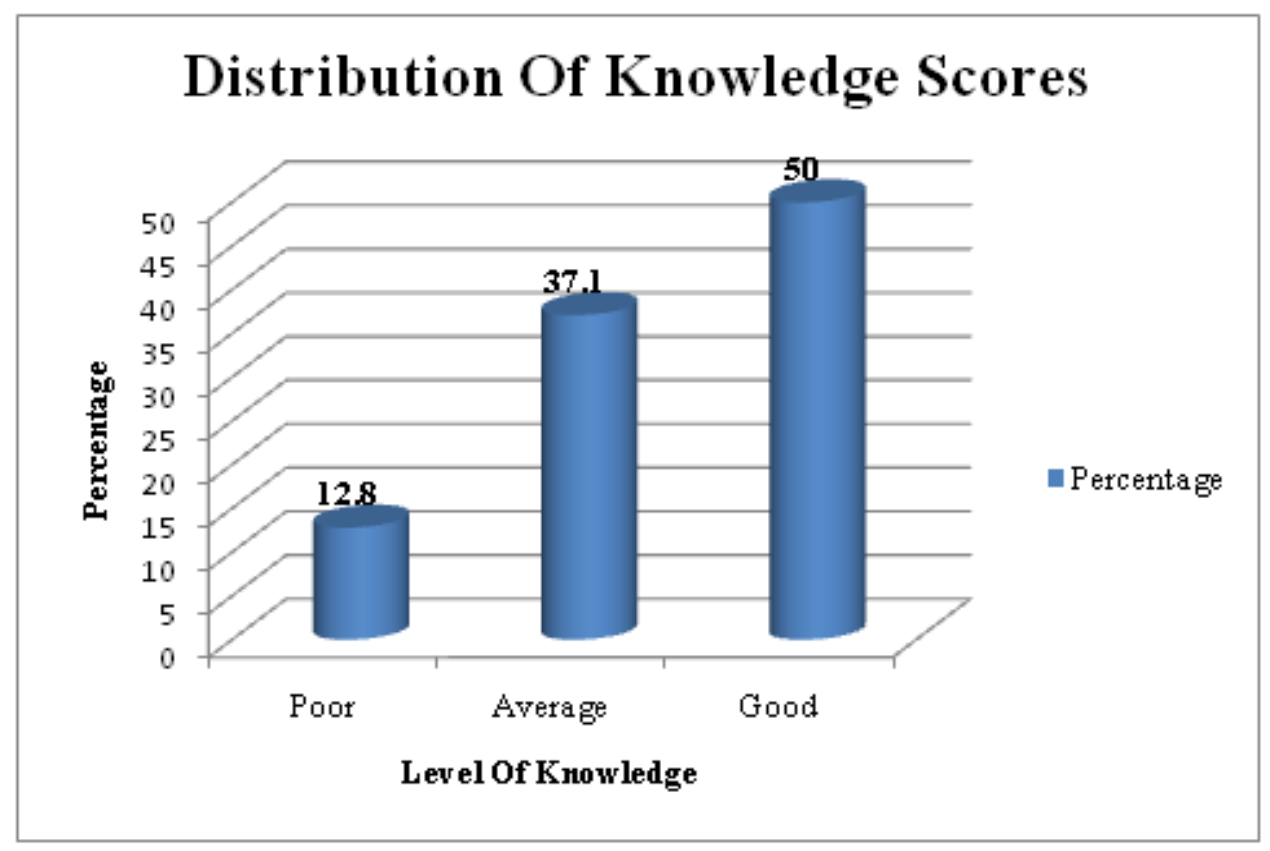

Fig - 1: Bar Diagram depicting the percentage distribution of knowledge scores

Level of association between nurses' knowledge regarding ECG interpretation during cardiac emergencies and their selected demographic variables

The study shows that there is a significant association of knowledge of nurses regarding ECG interpretation during cardiac emergencies with their selected demographic variables such as present departmental experience and years of experience. The remaining demographic variables, such as age, gender, professional qualification and exposure to ECG training programme have no association with the knowledge level of the nurses.

\section{CONCLUSION}

The study concluded that $12.8 \%$ of the staff nurses had poor knowledge, $37.1 \%$ of them had an average knowledge and $50 \%$ of them had a good knowledge regarding ECG interpretation during cardiac emergencies. Also there is a significant association between knowledge level of cardiac nurses regarding ECG interpretation during cardiac emergencies and their selected demographic variables such as present departmental experience and years of experience and the remaining variables had no such association. 


\section{ARTICLES}

\section{IMPLICATIONS OF THE STUDY}

The findings of the study have implications for Nursing Education, Nursing Practice, Nursing Research and Nursing administration.

\section{The findings of this study have scope in the following areas -}

\section{Nursing Education:}

- The present study emphasises on the encouragement of staff nurses to undergo continuing nursing education programme, specialised courses or training regarding cardiac diseases. There should be an ongoing feedback on the performance of the nurses in providing care to the cardiac patients.

- The knowledge among student nurses should be disseminated through the emphasis on planned clinical teaching and incidental teaching during their hospital postings in relation to the care of cardiac patients.

\section{Nursing Practice:}

The study shows varied degrees of knowledge deficit among the staff nurses regarding care of patients with cardiac disorders.

- The study shows the need for correction of the knowledge deficiency among the nurses.

- Nurses working in cardiac hospital should be encouraged to have continuing education and also through inservice education and their competency has to be measured by performance appraisal.

- The study also highlights the need to develop protocols and nursing standards in caring for the patients with cardiac emergencies.

\section{Nursing Administration:}

- Nursing administrator should be provided with manpower, money and material in planning and arranging educational sessions and workshops by subject experts to the nursing personnel to update their knowledge.

- The ward-in-charge should be encouraged to strengthen their supervising skills, which will enable them to guide the new staff to have competent knowledge.

- The nursing superintendent should arrange for educational sessions and workshops by the subject experts.

\section{Nursing Research:}

- As research helps in broadening the nursing horizons, the present study has given the base to conduct in future quantitative as well as qualitative researches on knowledge of staff nurses regarding care for the patients with cardiac disorders.

- Use of research findings becomes a quality assurance evaluation tool to enhance individual performance as a whole. 


\section{ARTICLES}

\section{REFERENCES}

1. AlGhatrif M \& Lindsay J (2012). A brief review: history to understand fundamentals of electrocardiography. Journal of community hospital internal medicine perspectives, 2(1), 143-83.

2. George A, Arumugham P S, \& Figueredo V M. (2010). AVR-the forgotten lead. Experimental \& clinical cardiology, 15(2), e36.

3. WHO (2017, January). The top 10 causes of death. Retrieved from: http://www.who.int/mediacentre/factsheets/fs310/en/

4. Hernández-Padilla J, Suthers F, Fernández-Sola C, \& Granero-Molina J (2016). Development and psychometric assessment of the basic resuscitation skills self-efficacy scale. European journal of cardiovascular nursing, 15(3), e10-e18.

5. Atwood D \& Wadlund D L (2015). ECG interpretation using the CRISP method: A guide for nurses. AORN journal, 102(4), 396-408.

6. Funk M, Fennie K P, Stephens K E, May J L, Winkler C G, Drew B. J. PULSE site investigators. (2017). Association of implementation of practice standards for electrocardiographic monitoring with nurses' knowledge, quality of care, and patient outcomes. Circulation: cardiovascular quality and outcomes, 10(2), e 3132.

7. Drew B J, \& Funk M. (2006). Practice standards for ECG monitoring in hospital settings: executive summary and guide for implementation, 157-168.

8. Larson T S, \& Brady W J. (2008). Electrocardiographic monitoring in the hospitalized patient: a diagnostic intervention of uncertain clinical impact. The American journal of emergency medicine, 26(9), 1047-1055.

9. Pottle A. (2005). A nurse-led rapid access chest pain clinic-experience from the first 3 years. European journal of cardiovascular nursing, 4(3), 227-233.

10. Funk M, Winkler C G, May J L, Stephens K, Fennie K P, Rose L \& Drew B J. (2010). Unnecessary arrhythmia monitoring and underutilization of ischemia and QT interval monitoring in current clinical practice: baseline results of the practical use of the latest standards for electrocardiography trial. Journal of electro cardiology, 43(6), 542-547.

11. Spiers C M. (2007). Using the 12-lead ECG to diagnose acute myocardial infarction in the presence of left bundle branch block. Accident and emergency nursing, 15(1), 56-61.

12. Benjamin E J, Blaha M J, Chiuve S E, Cushman M, Das S R, Deo R \& Jiménez M C.(2017). Heart disease and stroke statistics-2017 update: a report from the American Heart Association. Circulation, 135(10), e146-e603.

13. Pathak L A, Shirodkar S, Ruparelia R, \& Rajebahadur J (2017). Coronary artery disease in women. Indian Heart Journal, 69(4), 532-538.

14. Gupta R, Puri V, Narain V V (1999). Cardiovascular risk profile in Indian women (Abstr). Indian Heart Journal; 51:679.

15. Refaey A S T (2012). Impact of designed teaching protocol about advanced cardiac life support (ACLS) on critical care nurse's knowledge and practices at Benha University Hospital, Cairo, Egypt. J Am Sci, 8(12), 838-50. 